\title{
A CRISPR view of development
}

\author{
Melissa M. Harrison, ${ }^{1}$ Brian V. Jenkins, ${ }^{2}$ Kate M. O'Connor-Giles, ${ }^{3,4}$ and Jill Wildonger ${ }^{2}$ \\ ${ }^{1}$ Department of Biomolecular Chemistry, University of Wisconsin School of Medicine and Public Health, Madison, Wisconsin \\ 53706, USA; ${ }^{2}$ Biochemistry Department, University of Wisconsin-Madison, Madison, Wisconsin 53706, USA; ${ }^{3}$ Laboratory \\ of Genetics, ${ }^{4}$ Laboratory of Cell and Molecular Biology, University of Wisconsin-Madison, Madison, Wisconsin 53706, USA
}

The CRISPR (clustered regularly interspaced short palindromic repeat)-Cas9 (CRISPR-associated nuclease 9) system is poised to transform developmental biology by providing a simple, efficient method to precisely manipulate the genome of virtually any developing organism. This RNA-guided nuclease (RGN)-based approach already has been effectively used to induce targeted mutations in multiple genes simultaneously, create conditional alleles, and generate endogenously tagged proteins. Illustrating the adaptability of RGNs, the genomes of $>20$ different plant and animal species as well as multiple cell lines and primary cells have been successfully modified. Here we review the current and potential uses of RGNs to investigate genome function during development.

Through the regulated process of development, a single cell divides and differentiates into the multitude of specialized cells that compose a mature organism. This process is controlled in large part by differential gene expression, which generates cells with distinct identities and phenotypes despite nearly identical genomes. Recent advances in genome engineering provide the opportunity to efficiently introduce almost any targeted modification in genomic DNA and, in so doing, the unprecedented ability to probe genome function during development in a diverse array of systems.

Over 25 years ago, homologous recombination (HR)based gene targeting in mice opened the door to targeted genome engineering for the study of development (Smithies et al. 1985; Thomas et al. 1986; Mansour et al. 1988). HR has been widely used in mice, but its low efficiency requires the selection of rare events in embryonic stem cells, and its implementation in most other organisms has been limited. In contrast, sequence-specific nucleases efficiently catalyze genome editing in a wide variety of organisms. Zinc finger nucleases (ZFNs), transcriptional activator-like effector nucleases (TALENs), and the recently developed two-component CRISPR (clustered regularly interspaced short palindromic repeat)Cas9 (CRISPR-associated nuclease 9) system can all be

[Keywords: CRISPR; Cas9; development; genome editing; genome engineering; RNA-guided nuclease]

Corresponding author: wildonger@wisc.edu

Article is online at http://www.genesdev.org/cgi/doi/10.1101/gad.248252.114.

Freely available online through the Genes \& Development Open Access option. programmed to generate targeted double-strand DNA breaks (DSBs) in genomic DNA. Researchers are able to co-opt the endogenous cellular pathways that repair these DSBs to introduce precise changes to the genome. The CRISPR-Cas9 system has propelled genome editing from being a technical possibility to a practical reality for developmental biology studies due to the simplicity with which the Cas9 nuclease is recruited to a specific DNA sequence by a small, easily generated guide RNA (gRNA) that recognizes its genomic target via standard Watson-Crick base-pairing. In this review, we highlight how this transformative technology is being exploited and applied to dissect developmental mechanisms in a wide variety of organisms and cultured cells.

\section{Making the cut: comparing the CRISPR-Cas9 system with ZFNs and TALENs}

DSBs induced by sequence-specific nucleases are most commonly repaired by the endogenous cellular DNA repair pathways of nonhomologous end joining (NHEJ) or homology-directed repair (HDR), both of which can be exploited to engineer the genome (Fig. 1). NHEJ is an error-prone process that involves direct ligation of the broken ends and can create disruptive insertions and deletions (indels) at targeted cleavage sites. The HDR pathway uses homologous DNA sequences as templates for repair, and, by supplying an exogenous repair template, HDR can be exploited to precisely edit genomic sequence or insert exogenous DNA.

Cas9 enzymes from type II CRISPR-Cas systems are emerging as the sequence-specific nucleases of choice for genome engineering for several reasons. Most notably, as an RNA-guided nuclease (RGN), Cas9 is guided by a single gRNA that is readily engineered. In the case of the most commonly used Cas9, derived from Streptococcus pyogenes, the gRNA targeting sequence comprises 20 nucleotides (nt) that can be ordered as a pair of oligonucleotides and rapidly cloned. In contrast, generating an effective ZFN or TALEN is labor-intensive (see Box 1). ZFNs and TALENs are proteins that combine uniquely designed and generated DNA-binding sequences with the FokI

\footnotetext{
(C) 2014 Harrison et al. This article, published in Genes \& Development, is available under a Creative Commons License (Attribution-NonCommercial 4.0 International), as described at http://creativecommons.org/ licenses/by-nc/4.0/.
} 
A

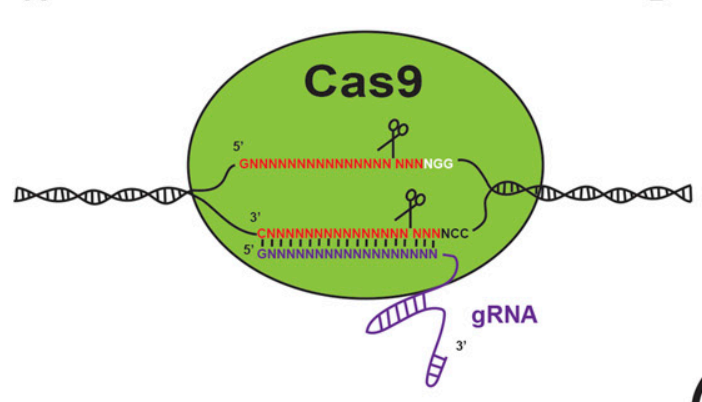

B

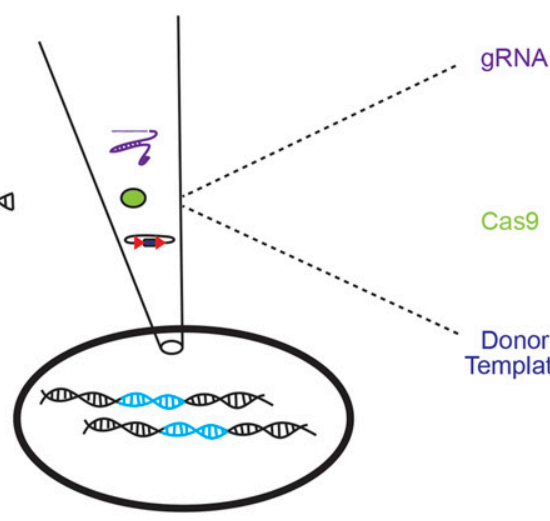

Component Delivery mechanism

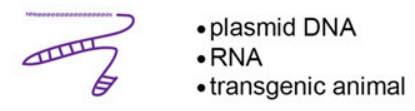

- transgenic animal

- plasmid DNA

- mRNA

- protein

-transgenic animal/cell
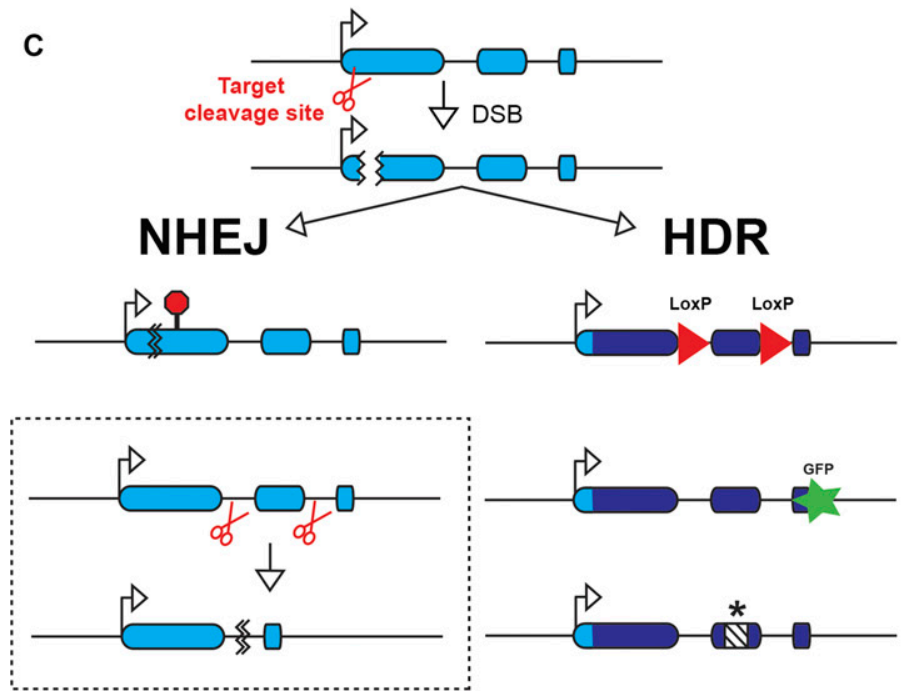
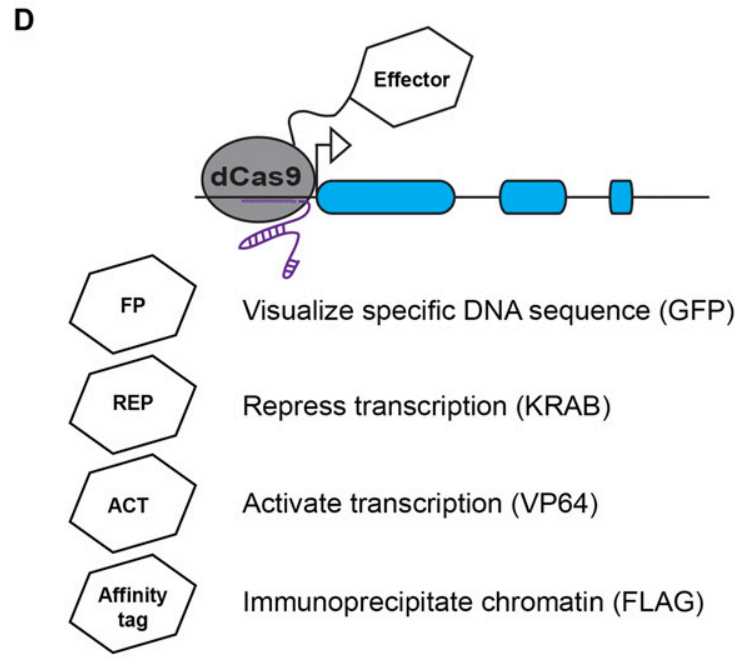

Repress transcription (KRAB)

Activate transcription (VP64)

Immunoprecipitate chromatin (FLAG)

Figure 1. The flexibility and adaptability of the CRISPR-Cas9 system offers vast potential for genome manipulations. (A) Overview of the CRISPR-Cas9 system. At its simplest, the system consists of the chimeric gRNA (purple), which guides the Cas9 nuclease to the genomic target site (red). The genomic target site is composed of 20 base pairs (bp) of homology with the gRNA (red) and a PAM sequence (white). Cleavage (scissors) occurs $3 \mathrm{bp} \mathrm{5'}$ of the PAM. (B) Components required for RGN-mediated genome editing. The CRISPR-Cas9 components can be delivered as DNA, RNA, or protein, as indicated, and introduced into the cell or embryo through injection, transfection, electroporation, or infection. Organisms and cells expressing transgenic Cas9 are available, and in Drosophila, both the transgenic Cas9-expressing strains and those expressing transgenic gRNA have been shown to increase targeting efficacy. To introduce designer mutations and/or exogenous sequence, a ssDNA or dsDNA donor template is included. $(C)$ Genome engineering outcomes. Cas9-induced DSBs can be repaired by either NHEJ or HDR. (Top left) The DSB generated by a single gRNA can be repaired by NHEJ to generate indels. (Bottom left, dashed box) With the use of two gRNAs, NHEJ can result in larger deletions. If the gRNAs target sequences on different chromosomes, it is possible to generate chromosomal translocations and inversions. (Right) With the inclusion of a researcher-designed donor template, HDR makes it possible to generate conditional alleles (top), fluorescently or epitopetagged proteins (middle), specific mutations (bottom), or any combination thereof. The donor template can also be designed to correct a mutation in the organism or cell or replace a gene. $(D)$ Catalytically inactive dCas9 provides a platform for probing genomic function. dCas9 can be fused to any number of different effectors to allow for the visualization of where specific DNA sequences localize, the repression or activation of transcription, or the immunoprecipitation of the bound chromatin.

nuclease cleavage domain. FokI is an obligate dimer, necessitating the generation of two novel proteins per editing experiment compared with a single gRNA for CRISPR-Cas9-mediated targeting.

The modular nature of the two-component CRISPRCas9 system and small size of the targeting gRNA have the added advantage of being particularly amenable to multiplexing. The use of the common Cas9 nuclease in conjunction with multiple gRNAs to introduce mutations in several genes simultaneously has been carried out in cultured mammalian cells as well as genetic model organisms such as mice, zebrafish, and Arabidopsis (Chang et al. 2013; Cong et al. 2013; Feng et al. 2013; Hwang et al. 2013; Li et al. 2013a; Mali et al. 2013c; Mao et al. 2013; Wang et al. 2013a). More recently, multiplexing was successfully used to introduce mutations in monkeys and silkworms (Wang et al. 2013b; Daimon et al. 2014; Niu et al. 2014b).

Finally, the CRISPR-Cas9 system couples efficiency and high specificity with minimal off-target effects when 


\section{Box: 1. A miniguide to genome engineering techniques}

Zinc finger nucleases (ZFNs), transcriptional activator-like effector nucleases (TALENs), and CRISPR (clustered regularly interspaced short palindromic repeat)-Cas9 (CRISPR-associated nuclease 9) all function on a similar principle: A nuclease is guided to a specific sequence within the genome to induce a doublestrand DNA break (DSB). Once a DSB is generated, the cell's intrinsic DNA repair machinery is set in motion, and it is during the repair of the DSB that the genome is modified. DSBs are typically repaired by either nonhomologous end joining (NHEJ) or homology-directed repair (HDR) (Fig. 1C). In NHEJ, the two cleaved ends of the DSB are ligated together. During this process, DNA of varying sizes, generally on the order of a few base pairs, is occasionally inserted and/or deleted randomly. When a DSB is targeted to a coding exon, these insertions or deletions (indels) can result in a truncated gene product. If two DSBs are induced, NHEJ can generate deletions, eliminating an entire gene or region. HDR uses homologous sequence as a template to repair the DSB. Researchers can take advantage of this repair pathway to introduce designer mutations or exogenous sequence, such as genetically encoded tags, by supplying the cell with a donor DNA template that has homology with the sequence flanking the DSB. Note that cells can also use endogenous DNA as a template, in which case the DSB is repaired without incorporation of the donor-supplied edits. It is important to keep in mind that although the researcher directs where the DSB occurs in the genome, the cell is in control of how the DSB is repaired, which determines the ultimate outcome of a genome-editing experiment.

\section{ZFNS}

ZFNs are fusion proteins comprised of DNA-binding $\mathrm{C}_{2} \mathrm{H}_{2}$ zinc fingers fused to the nonspecific DNA cleavage domain of the nuclease Fok1 (for review, see Carroll 2011). Each zinc finger can be engineered to recognize a nucleotide triplet, and multiple (typically three to six) zinc fingers are joined in tandem to target specific genome sequences. Because the Fok1 cleavage domain must dimerize to be active, two ZFNs are required to create a DSB. This technique, which was first successfully used in fruit flies more than a decade ago (Bibikova et al. 2002), has since been used to modify the genomes of many different organisms, including those that had not previously been developed as genetic model systems.

\section{TALENS}

Similar to ZFNs, TALENs are chimeric proteins comprised of a programmable DNA-binding domain fused to the Fok1 nuclease domain (for review, see Joung and Sander 2013). TALEs are naturally occurring proteins that are secreted by the bacteria Xanthamonas and bind to sequences in the host plant genome, activating transcription. The TALE DNAbinding domain is composed of multiple repeats, each of which are 33-35 amino acids long. Each repeat recognizes a single nucleotide in the target DNA sequence. Nucleotide specificity is conferred by a two-amino-acid hypervariable region present in each repeat. Sequence-specific TALENs are generated by modifying the two residues in the hypervariable region and concatenating multiple TALE repeats together. Because the TALE DNA-binding domain is fused to Fok1, TALENs, like ZFNs, must also be used as dimers to generate DSBs.

\section{The CRISPR-Cas9 system}

The CRISPR-Cas9 genome-editing method is derived from a prokaryotic RNA-guided defense system (Gasiunas et al. 2012; Jinek et al. 2012, 2013; Cong et al. 2013; Mali et al. 2013c). CRISPR repeats were first discovered in the Escherichia coli genome as an unusual repeat locus (Ishino et al. 1987). The significance of this structure was appreciated later when investigators realized that phage and plasmid sequences are similar to the spacer sequences in CRISPR loci (Bolotin et al. 2005; Mojica et al. 2005; Pourcel et al. 2005). Soon afterward, it was shown that spacers are derived from viral genomic sequence (Barrangou et al. 2007).

In the CRISPR-Cas system, short sequences (referred to as "protospacers") from an invading viral genome are copied as "spacers" between repetitive sequences in the CRISPR locus of the host genome. The CRISPR locus is transcribed and processed into short CRISPR RNAs (crRNAs) that guide the Cas to the complementary genomic target sequence. There are at least eleven different CRISPRCas systems, which have been grouped into three major types (I-III). In the type I and II systems, nucleotides adjacent to the protospacer in the targeted genome comprise the protospacer adjacent motif (PAM). The PAM is essential for Cas to cleave its target DNA, enabling the CRISPR-Cas system to differentiate between the invading viral genome and the CRISPR locus in the host genome, which does not incorporate the PAM. For additional details on this fascinating prokaryotic adaptive immune response, see recent reviews (Sorek et al. 2013; Terns and Terns 2014).

Type II CRISPR-Cas systems have been adapted as a genome-engineering tool. In this system, crRNA teams up with a second RNA, called trans-acting CRISPR RNA (tracrRNA), which is critical for crRNA maturation and recruiting the Cas9 nuclease to DNA (Deltcheva et al. 2011; Jinek et al. 2012). The RNA that guides Cas9 uses a short ( 20-nt) sequence to identify its genomic target. This three-component system was simplified by fusing together crRNA and tracrRNA, creating a single chimeric "guide" RNA (abbreviated as sgRNA or simply gRNA) (Gasiunas et al. 2012; Jinek et al. 2012). While some early experiments indicated that a gRNA may not cleave a subset of targets as efficiently as a crRNA in combination with tracrRNA (Mali et al. 2013c), the ease of using a single RNA has led to the widespread adoption of gRNAs for genome engineering. A number of resources for designing experiments using the CRISPR-Cas9 system are freely available online. (A comprehensive list is available at http://www. geewisc.wisc.edu.)
gRNAs are well designed. This was evident in wholegenome analysis of engineered human stem cells from multiple laboratories, which uncovered few off-target effects (Duan et al. 2014; Kiskinis et al. 2014; Smith et al. 2014; Suzuki et al. 2014; Veres et al. 2014). Recent work has shown that truncating the $5^{\prime}$ end of the gRNA, where mismatches with the genomic target sequence are tolerated, further increases specificity (Fu et al. 2014). The potential for off-target cleavage can also be reduced by using a mutant nickase version of Cas9 (Cong et al. 
2013; Mali et al. 2013a; Ran et al. 2013; Chen et al. 2014; Cho et al. 2014; Fauser et al. 2014; Fujii et al. 2014; Lin et al. 2014; Rong et al. 2014; Shen et al. 2014). In this approach, pairs of Cas9 nickases are targeted to generate single-strand breaks on opposite strands of the genomic target DNA. While the frequency of off-target effects is also low for TALENs, off-target cleavage has been a significant concern for ZFNs (for review, see Carroll 2014). The coassembly of multiple zinc fingers in a single ZFN can alter how the zinc finger and DNA interact, reducing specificity and posing challenges for optimal ZFN design. It is of interest to note that rare-cleaving meganucleases (also known as homing endonucleases) hold the promise of even greater specificity than TALENs or RGNs, which makes them of great interest for gene therapy applications. A drawback of meganucleases, however, has been in the difficulty of programming the nuclease to recognize a desired target. To circumvent this issue, a recent study has generated a meganuclease-TALE chimera (megaTAL), which uses the TALE DNA-binding module to target a meganuclease to the desired sequence in the genome (Boissel et al. 2014). Analogous efforts to combine the precision of meganucleases with the flexibility and ease of targeting of RGNs may further revolutionize genome engineering.

\section{Practical considerations of using RGNs in developing organisms}

RGNs hold great potential for dissecting how the genome functions during development. Since the CRISPR-Cas9 system has been recently described in detail elsewhere (Hsu et al. 2014; Sander and Joung 2014), we provide just a brief overview of the system (Box 1; Fig. 1A-C) and focus here on a few practical considerations for using RGNs to edit the genome of a developing organism.

The current methods of producing the CRISPR-Cas9 components provide great flexibility in terms of expression and delivery, and biologists can exploit these options to control when and where DSBs are generated in an organism. To introduce DSBs and generate modifications early in development, the CRISPR-Cas9 components can be injected as DNA, RNA, or protein into most developing organisms. This approach, which has been widely used, generates mosaic organisms for analysis. To gain control over which tissues are affected, a plasmid expressing Cas9 under the control of tissue-specific enhancers can be used. Since each cell has a choice of whether to repair a break through NHEJ or HDR, a variety of different repair events will be present in the injected organism (and in individual cells). The frequency at which both alleles of a gene are affected has been reported to be high enough to visualize null phenotypes in developing mice and zebrafish (Jao et al. 2013; Wang et al. 2013a; Yasue et al. 2014; Yen et al. 2014). For example, zebrafish and mice injected with a gRNA targeting the tyrosinase gene resulted in embryos displaying mosaic pigmentation (Jao et al. 2013; Yen et al. 2014). This indicates that it is possible to generate homozygous mutant tissue in developing animals through injection of the CRISPR-Cas9 components.
Genetic mosaicism, however, may not be desirable for all experiments. In this case, it is necessary to first generate a strain that has the desired modifications. Thus, the targeted modification must occur in the germ cells of the injected organism and be compatible with viable progeny. In fruit flies, this process is facilitated by using transgenic animals that selectively express Cas9 in the germline (Kondo and Ueda 2013; Ren et al. 2013; Gratz et al. 2014; Sebo et al. 2014; Xue et al. 2014). The frequency of targeted events is increased using Cas9 transgenic animals, most likely because more consistent levels of Cas9 are achieved with a stably integrated transgene than with injected plasmid, mRNA, or protein. Transgenic expression of the gRNA has also been demonstrated to increase the frequency of targeted events in fruit flies (Kondo and Ueda 2013; Port et al. 2014; Xue et al. 2014). The expression of Cas9 can be restricted to the germline by placing it under the control of tissuespecific regulatory sequences. In contrast, gRNAs are expressed using polymerase III (Pol III) promoters, which are used to prevent gRNAs from being unduly modified and exported from the nucleus. However, Pol III promoters are typically constitutively active and lead to the ubiquitous expression of gRNAs. To provide spatial and temporal control of gRNA expression, strategies to place gRNAs under the control of Pol II promoters are being developed (Gao and Zhao 2014; Nissim et al. 2014). One approach includes nesting gRNA sequence between ribozymes, thus liberating the gRNA from a Pol II transcript. Other approaches take advantage of the endoribonuclease Csy4, a CRISPR-associated enzyme also known as Cas6, which processes CRISPR repeats and can free an appropriately sized gRNA from cellular transcripts (Nissim et al. 2014; Tsai et al. 2014). These approaches will allow greater control over gRNA expression in developing organisms and in cultured cells and would also enable multiple gRNAs to be generated from a single transcript.

Genome engineering with RGNs enables the direct manipulation of nearly any sequence in the genome to determine its role in development. The major limitation as to which genomic loci can be targeted is the requirement of a specific protospacer adjacent motif (PAM). The PAM is a short DNA motif adjacent to the Cas9 recognition sequence in the target DNA and is essential for cleavage. The most commonly used $S$. pyogenes Cas9 requires the PAM sequence $5^{\prime}$-NGG (in cell lines, other PAMs are recognized, including $5^{\prime}-\mathrm{NAG}$, but at a lower frequency) (Jinek et al. 2012; Esvelt et al. 2013; Hsu et al. 2013; Jiang et al. 2013a; Zhang et al. 2014). The PAM is critical for cleavage and increases target specificity but, conversely, can also make some segments of the genome refractory to Cas9 cleavage. For example, AT-rich genomic sequences may contain fewer PAM sites that would be recognized and cleaved by $S$. pyogenes Cas9. Thus, some poly(dA-dT) tracts, which are implicated in nucleosome positioning (for review, see Struhl and Segal 2013), may be difficult to manipulate using $S$. pyogenes Cas9. However, Cas9 from other bacteria recognizes different PAM sequences, and some have already been 
adapted for genome engineering in human cells. This includes Cas9 from Neisseria meningitides (5'-NNNN GATT), Streptococcus thermophilus (5'-NNAGAAW), and Treponema denticola (5'-NAAAAC) (Cong et al. 2013; Esvelt et al. 2013; Hou et al. 2013). These nucleases extend the catalog of possible target sequences, opening up different parts of a genome to analysis. Furthermore, Cas9 from different bacteria can be used in combination, increasing the multiplex capabilities of the CRISPR-Cas9 system.

\section{Catching a break with RGNs to introduce genome modifications}

With RGNs, a variety of genomic manipulations are brought within reach of developmental biologists studying a diversity of organisms (Table 1). This approach also makes it possible to readily generate mutations in different genetic strains, making it easier to control genetic background and eliminating the need to carry out multigenerational mating schemes to bring different mutations together in the same animal. While the CRISPR-Cas9 system has been widely used to introduce indels and deletions, HDR makes it possible to introduce more precise gene mutations, deletions, and exogenous sequences, such as loxP sites and green fluorescent protein (GFP).

\section{Multiplexing advantages}

Genes that have essential roles in development are often functionally redundant, and thus the effects of mutating a single gene can be masked by the presence of another gene. Due to the ease and efficiency with which gRNAs can be generated, multiple gRNAs can be used in a single experiment to simultaneously mutate multiple genes, overcoming issues of redundancy. Recent technical innovations now make it possible to express multiple gRNAs from a single transcript (Nissim et al. 2014; Tsai et al. 2014), making RGN multiplexing experiments even easier to carry out. Such multiplexing experiments will also facilitate multifaceted experiments, including epistasis tests and manipulating genes that are physically very close together in the genome. Multiplexing has already been used successfully to simultaneously disrupt both Tet 1 and Tet2 in developing mice following injection into zygotes (Wang et al. 2013a). The CRISPRCas9 system has also been used to eliminate two genes in monkeys (Niu et al. 2014b), demonstrating the potential

Table 1. Organisms that have been modified using the CRISPR-Cas9 system

\begin{tabular}{|c|c|c|c|c|c|}
\hline \multirow[b]{3}{*}{ Organism } & \multicolumn{2}{|c|}{ Mutations created in } & \multirow{2}{*}{\multicolumn{2}{|c|}{ Alleles generated by }} & \multirow[b]{3}{*}{ References } \\
\hline & \multirow{2}{*}{$\begin{array}{l}\text { Cultured } \\
\text { cells }\end{array}$} & \multirow{2}{*}{$\begin{array}{l}\text { Organism } \\
\text { (heritable?) }\end{array}$} & & & \\
\hline & & & NHEJ & HDR & \\
\hline \multicolumn{6}{|l|}{ Vertebrates } \\
\hline Axolotl & & $\boldsymbol{r}$ & $\boldsymbol{}$ & & Flowers et al. 2014 \\
\hline Frog & & $\boldsymbol{\nu}($ Yes $)$ & レ & & Blitz et al. 2013; Nakayama et al. 2013; Guo et al. 2014 \\
\hline Human & $\boldsymbol{}$ & & レ & レ & For review, see Sander and Joung 2014 \\
\hline Medaka & & レ(Yes) & レ & & Ansai and Kinoshita 2014 \\
\hline Mouse & $\boldsymbol{\nu}$ & $\boldsymbol{レ}($ Yes) & $\boldsymbol{}$ & $\boldsymbol{}$ & For review, see Sander and Joung 2014 \\
\hline Monkey & & レ & レ & & Niu et al. $2014 b$ \\
\hline Pig & レ & $\boldsymbol{\nu}($ Yes $)$ & レ & & Hai et al. 2014; Sato et al. 2014 \\
\hline Rabbit & & $\nu$ & レ & & Yang et al. 2014 \\
\hline Rat & $\boldsymbol{}$ & $\boldsymbol{\nu}($ Yes $)$ & レ & レ & Li et al. 2013a,b, 2014b; Ma et al. 2014b,c,d \\
\hline Tilapia & & $\boldsymbol{\nu}($ Yes $)$ & レ & & Li et al. $2014 \mathrm{a}$ \\
\hline Zebrafish & & $\boldsymbol{レ}$ (Yes) & レ & レ & For review, see Auer et al. 2014 \\
\hline \multicolumn{6}{|l|}{ Invertebrates } \\
\hline Freshwater flea & & $\boldsymbol{\nu}($ Yes $)$ & レ & & Nakanishi et al. 2014 \\
\hline Fruit fly & レ & $\boldsymbol{\nu}$ (Yes) & レ & レ & For review, see Gratz et al. 2013; Bassett and Liu 2014 \\
\hline Roundworm & & $\boldsymbol{\nu}($ Yes $)$ & レ & レ & For review, see Waaijers and Boxem 2014 \\
\hline Silkworm & レ & $\boldsymbol{\nu}($ Yes $)$ & レ & レ & $\begin{array}{l}\text { Wang et al. 2013b; Daimon et al. 2014; } \\
\text { Liu et al. 2014b; Ma et al. 2014a; Wei et al. } 2014\end{array}$ \\
\hline \multicolumn{6}{|r|}{ 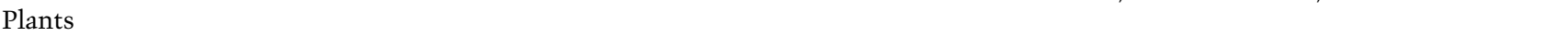 } \\
\hline Corn & & レ & レ & & Liang et al. 2014 \\
\hline Liverwort & & $\boldsymbol{\nu}($ Yes $)$ & レ & & Sugano et al. 2014 \\
\hline Rice & & $\boldsymbol{\nu}($ Yes $)$ & $\boldsymbol{\nu}$ & & For review, see Belhaj et al. 2013 \\
\hline Sorghum & & レ & レ & & Jiang et al. $2013 \mathrm{~b}$ \\
\hline Sweet orange & & & レ & & Jia and Wang 2014 \\
\hline Thale cress & & $\boldsymbol{\nu}($ Yes $)$ & レ & レ & For review, see Belhaj et al. 2013 \\
\hline Tobacco & & $\boldsymbol{レ}$ (Yes) & レ & 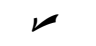 & For review, see Belhaj et al. 2013 \\
\hline Wheat & $\boldsymbol{\nu}$ & & レ & & Upadhyay et al. 2013 \\
\hline
\end{tabular}

We limited our list to those organisms that provide platforms for the study of development (as indicated for some organisms, only cells derived from the organism have been modified to date). Blanks indicate "not tested." For the organisms in which the CRISPR-Cas9 system has been used extensively, see recent reviews. Given the rapid advances in the field, we apologize for any organisms or references that were inadvertently not included. 
of RGN-mediated mutagenesis to create primate models of multigenic human disorders. Multiplexing can be further extended by taking advantage of a catalytically inactive Cas9 that has been adapted as a programmable RNAguided platform to regulate gene expression (see below) and by using Cas9 from different bacteria in combination.

\section{Conditional alleles}

Many gene products of interest to developmental biologists are essential early in development, and mutations in these genes are lethal to an animal before it reaches later developmental stages. Conditional alleles provide spatial and temporal control over gene inactivation and therefore have been invaluable tools for working with genes that cause early lethality. Conditional alleles have also been used to determine where and when a gene is acting during development. The utility of exerting conditional control over gene activity is widely recognized, and an international consortium is currently working to create a library of conditional alleles for $\sim 20,000$ genes in the mouse genome (Skarnes et al. 2011). While conditional alleles are typically created using $\mathrm{HR}$, in the past year, RGNs have been used to rapidly generate conditional alleles in mice, rats, and flies (Yang et al. 2013; Gratz et al. 2014; Ma et al. 2014d). RGNs will also be valuable in expanding the repertoire of recombinase-expressing lines. Since the expression of the conditional allele reflects the expression pattern of the recombinase, it is advantageous to have a variety of lines that express recombinase in specific tissues or at discrete developmental stages. The CRISPRCas9 system was recently used to generate two different Cre recombinase-expressing lines in rats (Ma et al. 2014b). Thus, RGNs are being used to rapidly generate the tools necessary to probe gene function in a tissue- and time-dependent manner.

\section{Introducing tags to illuminate and manipulate development}

Simply observing an organism as it develops can yield valuable insight into mechanisms that regulate processes such as cell proliferation, differentiation, and morphogenesis and tissue growth. At the cell and molecular level, a broad selection of molecular tags has enabled researchers to track the localization of proteins and RNAs in fixed and live tissue during development. While endogenous genes can be tagged using HR or transposable elements (TEs), these approaches are generally technically challenging and time-consuming. Thus, localization experiments often use transgenes to ectopically express tagged proteins or RNAs. However, a drawback of this approach is that transgenes are typically expressed using exogenous promoters and/or enhancers and are integrated in the genome in a nonnative chromatin environment. Therefore, transgenes are often misexpressed relative to the wild-type gene, and this difference in expression pattern or level can result in altered localization and/or activity of the tagged protein or RNA. A key advantage of tagging an endogenous gene is eliminating expression artifacts. A limitation of this approach is that natively expressed protein or RNA may not be easily detected if it is weakly expressed and/or dispersed within cells. To overcome difficulties resulting from sparse expression, approaches such as tag multimerization may be necessary to effectively visualize endogenous proteins and RNAs.

RGNs open the door to quickly and easily tagging endogenous genes for developmental studies. Furthermore, because the CRISPR-Cas9 system is amenable to multiplexing, tags could be added simultaneously to multiple genes or different splice isoforms of a single gene. There is an ever-growing number of genetically encoded molecular tags that can be used for functional analysis, protein purification, or protein and RNA localization studies.

Tagging proteins and RNAs to visualize their expression in vivo is likely to be a common application of RGNs. Indeed, groups working with flies, mice, and rats have already used epitope tags and fluorescent proteins to label endogenous proteins and generated gene expression reporters (Yang et al. 2013; Ma et al. 2014b; Yu et al. 2014). In flies, a histone acetyltransferase protein encoded by the gene chameau was C-terminally tagged with GFP, and myc was used to tag an uncharacterized gene, CG4221 (Yu et al. 2013). In mice, the Sox2 gene was tagged with the V5 epitope (Yang et al. 2013). Additionally, two different fluorescent reporters were generated for the genes nanog and Oct4 (Yang et al. 2013). These reporters used either the viral $2 \mathrm{~A}$ peptide or an internal ribosome entry site (IRES) to express fluorescent proteins with the same expression pattern as the endogenous gene but not fused to the protein product. While these groups used standard fluorescent proteins, a spectrum of fluorescent proteins of different colors and with diverse functions are available (for review, see Dean and Palmer 2014).

In addition to tags for visualizing protein localization, there are also genetically encoded tags to manipulate other aspects of protein function. The CRISPR-Cas9 system was recently used to introduce a small destabilization domain tag into the Treacher Collins-Franceschetti syndrome 1 (TCOF1) gene in human 293T cells (Park et al. 2014). This FKBP protein-based destabilization tag makes the protein to which it is attached susceptible to degradation in the absence of an exogenously supplied chemical, enabling precise post-transcriptional control over protein expression (Banaszynski et al. 2006). As the RGN-mediated tagging of endogenous proteins becomes more widely adapted, it will be interesting to see how this will drive an expansion of the current repertoire of genetically encoded fluorescent proteins and tags.

\section{Dishing up cultured cells with modified genomes}

Cultured cells are an important and widely used system for dissecting cellular, molecular, and biochemical mechanisms of development. Moreover, human-derived cells are essential for characterizing the function of human genes that lack orthologs in other organisms. The first reports of genome engineering using RGNs were carried out in cultured mammalian cells (Cong et al. 2013; Jinek et al. 2013; Mali et al. 2013c), and, in the short time since 
these initial reports, RGNs have been used successfully in a large number of cell lines. Mammalian cell lines, such as HEK293 cells, are relatively easy to manipulate and have been extensively used as a quick and straightforward system to characterize and develop rapid improvements in the technology. Because there have been a number of recent reviews covering the use of RGNs (Mali et al. 2013b; Hsu et al. 2014; Wilkinson and Wiedenheft 2014), we focus here on applications of particular interest for the study of developmental mechanisms, specifically the ability to engineer the genomes of pluripotent stem cells.

Human pluripotent stem cells have been difficult to engineer using classic gene targeting strategies (Capecchi 2005; Hockemeyer and Jaenisch 2010). New avenues of genetic manipulation of these cell types have been opened up by RGNs, which can be readily programmed with different gRNAs. One of the first reports of the use of RGNs for genome engineering demonstrated success in induced pluripotent stem cells (iPSCs) with a frequency of between $2 \%$ and $4 \%$ when assayed by deep sequencing of bulk culture (Mali et al. 2013c). Recovery of engineered cells is increased when Cas9-expressing cells are marked with a fluorescent marker and selected by cell sorting (Ding et al. 2013). Using this strategy, it was reported that clones containing at least one mutant allele could be isolated at frequencies between $51 \%$ and $79 \%$. In comparison, TALENs designed against the same set of genes resulted in between $0 \%$ and $34 \%$ of clones containing at least one mutant allele. This use of RGNs resulted in a considerable improvement over ZFNs and TALENs in the efficiency of genome engineering in pluripotent cells.

To further increase the frequency of editing events and eliminate the necessity for cell sorting, three human PSC (hPSC) lines and one iPSC line were generated that express doxycycline-inducible Cas9 (iCRISPR) (Gonzalez et al. 2014). Because gRNAs are relatively small $(\sim 100$ $\mathrm{nt})$, these RNAs can be transfected into cell types that are poorly transfectable, such as hPSCs. This system has been used for multiplexing and to generate biallelic knockouts for six genes with efficiencies ranging from $17 \%$ to $67 \%$. Mutations could be generated after inducing differentiation, allowing for studies of tissue-specific effects. Pluripotent lines have also been made that enable the simultaneous induction of Cas9 and gRNA expression. These inducible pluripotent cell lines will enable the study of gene knockdown following differentiation into a wide variety of cell types.

As discussed below, the development of additional Cas9mediated tools for regulating gene expression in combination with lentiviral delivery systems has enabled the modulation of the differentiation status of hPSCs (Kearns et al. 2014). This continuously expanding Cas9-mediated toolbox will advance the study of genetic contributions to differentiation and disease mechanisms using pluripotent cells and has potential for use in gene therapy.

\section{Disease modeling and treatment with RGNs}

Gene editing provided by RGNs has offered and will continue to offer important advancements in genetic studies of disease. The ability to introduce deletions as well as point mutations in a wide variety of cell types allows for the generation of cell lines with human disease-causing mutations. A large number of genes in a diversity of human cell types have already been modified (for review, see Niu et al. 2014a), providing the capacity to study mechanistic effects of these mutations. The relative ease of generating mutant animals will yield many additional animal models of disease and supply a means of testing whether specific polymorphisms are the proximal cause of disease in vivo. Additionally, the CRISPR-Cas9 system is amenable to application in organisms not widely used for genetic studies. Organisms that may be better suited to mimic human disease can now be more easily used to generate disease models. For example, mouse models of the bleeding disorder von Willebrand disease fail to fully recapitulate the human disease. To address this, RGNs were used in pigs to engineer a knockout of the $V W F$ gene, whose deficiency causes the human disease (Hai et al. 2014). Because the organ size and vasculature of pigs more closely resemble those of humans as compared with the more commonly used mouse models, it is likely that studies in this model will better recapitulate the human disease state.

Apart from point mutations and gene deletions, large chromosomal rearrangements can drive specific cancers. By simultaneously introducing gRNAs targeting two different chromosomes or two widely separated regions of the same chromosome, RGNs have been used to introduce targeted inversions and translocations into otherwise wild-type human cells (Choi and Meyerson 2014; Torres et al. 2014). These engineered cells will ultimately allow for studies of the causative role of these gene fusions in cancer progression. Translocations that drive lung adenocarcinoma (Choi and Meyerson 2014), acute myeloid leukemia, and Ewing's sarcoma (Torres et al. 2014) have been generated in both HEK293 cells and more physiologically relevant cell types (nontransformed immortalized lung epithelial cells and human mesenchymal stem cells). Additionally, cell lines harboring chromosomal inversions found in lung adenocarcinoma have also been created (Choi and Meyerson 2014).

As genome editing with RGNs becomes increasingly efficient and precise, it provides exciting opportunities for gene therapy. Previous work using ZFNs and TALENs has demonstrated the promise suggested by genome editing for gene therapy (for review, see Lisa Li et al. 2014). Proof of principle for the utility of the CRISPR-Cas9 system has recently been shown for a number of well-characterized disease genes. The CRISPR-Cas9 system has been used to correct a mutation that causes cystic fibrosis in both patient-derived primary cultured small intestinal cells and large intestinal stem cells. When assayed in organoid culture, disease-associated defects were rescued in these engineered cells (Schwank et al. 2013). As discussed above, RGNs can also be used to edit the genomes of pluripotent cells such as iPSCs, allowing for the generation of cell type-specific disease models. Furthermore, such studies suggest that, in the future, it may be possible to generate iPSCs from patients, correct the causative 
mutation, and reintroduce these cells (or the differentiated derivatives of these cells) back into the patient to provide rescuing wild-type function.

Corrections of disease loci have also been generated in animals. RGNs were used in mouse embryos to correct a dominant mutation in the Crygc gene, which causes cataracts, and those mice with the corrected locus were free of cataracts (Wu et al. 2013). Providing additional promise for the potential of gene therapy in adults, delivery of the CRISPR-Cas9 system and a single-stranded donor template by hydrodynamic injection into mice was able to produce edited hepatocytes that were corrected for a point mutation in Fah, a gene that, when mutated, causes hereditary tyrosinemia. These hepatocytes were rescued for function, as indicated by the fact that resulting animals had substantially decreased liver damage as compared with Fah mutant mice (Yin et al. 2014). The adaptability of the CRISPR-Cas9 system and its high efficiency will result in significant progress in the mechanistic studies of disease and provide promise for advancements in gene therapies.

\section{Bringing surgical precision to genetic screens}

Advances in developmental biology have been propelled by genetic screens carried out in a variety of organisms and cultured cells. Genetic screens have used a number of different approaches to introduce mutations into the genome or knock down gene activity. These methods include the use of chemicals (e.g., alkylating agents such as EMS and ENU), TEs, and RNAi. Each of these methods has advantages and disadvantages; for example, although chemical mutagenesis generates unbiased mutations, these mutations are typically single base-pair changes and can be difficult (or sometimes impossible) to specifically identify. In contrast, TE-induced mutations are easier to map (for review, see Friedel and Soriano 2010; Ammar et al. 2012; St Johnston 2013). However, TEs often preferentially insert at particular sites, leading to TE "hot spots" and the lack of mutations in other genomic regions. This uneven distribution of TE-induced mutations can be somewhat addressed by using multiple TEs with different insertional preferences. Another caveat is that TEs can insert and excise multiple times before mapping. This can result in "hit-and-run" mutations for TEs that excise imprecisely, and these mutations can be difficult to identify. More recently, RNAi has been widely used for large-scale genetic screens in a variety of organisms and cultured cells (for review, see Mohr et al. 2010). Since base-pair interactions mediate target identification, the gene product targeted by RNAi is easy to determine based on sequence. Moreover, RNAi can be controlled temporally and spatially during development, a boon for characterizing genes that might cause early lethality and identifying the cells in which a gene is active. In addition, unlike conditional alleles, RNAi is reversible, allowing for screens to specifically target distinct cell populations at discrete developmental time points. Although RNAi has been a powerful tool for both forward and reverse genetic screens, there are some drawbacks to this approach: Off-target effects are common, targets are generally limited to mRNAs, and, since the target gene is not directly affected, expression is knocked down but not entirely eliminated.

RGN-based screens have the potential to combine the strengths of chemical and TE mutagenesis with the advantages of RNAi. Like RNAi, RGN targets are identified via standard base-pair interactions, making it relatively straightforward to predict which genes will be affected by a given gRNA. Because RGNs directly target genomic DNA, it is possible to target nearly every part of the genome, including noncoding RNAs that are difficult to target using RNAi. RGNs can also be designed to target short sequence motifs that are impossible to target with precision using TEs or chemical mutagenesis. Thus, RGN-based screens can be designed to systematically probe elements that, for example, regulate gene expression (promoters and enhancers) as well as motifs that direct the post-transcriptional processing of a transcript (splice site sequences and mRNA $5^{\prime}$ and $3^{\prime}$ untranslated regions). As described elsewhere in this review, a variety of different alleles can be generated using RGNs, and, furthermore, a catalytically inactive Cas9 can be used as a programmable platform for manipulating gene expression levels, expanding the repertoire of screening approaches.

The small size of gRNAs and the ease with which they are generated make it feasible to generate gRNA libraries for large-scale genetic screens. In fact, the first RGNbased genetic screens were recently carried out in cultured mammalian cells (Koike-Yusa et al. 2014; Shalem et al. 2014; Wang et al. 2014; Zhou et al. 2014). When carrying out such a screen, it is important to consider both the number of genes targeted by the library and the degree of coverage of each gene. The largest library reported to date is comprised of 90,000 gRNAs designed to target 19,000 genes, which equates to about four to five gRNAs per targeted gene (Koike-Yusa et al. 2014).The screens identified targets affecting the DNA mismatch repair pathway (Koike-Yusa et al. 2014; Wang et al. 2014), resistance to bacterial and chemical toxins (Koike-Yusa et al. 2014; Wang et al. 2014; Zhou et al. 2014), and cell survival and proliferation (Shalem et al. 2014; Wang et al. 2014). The Zheng group (Shalem et al. 2014) also compared the results of their screen for genes involved in resistance to a drug that inhibits B-Raf with a prior RNAi screen that used the same cell line and drug. This comparison revealed that gRNAs identified targets that could be validated more consistently and efficiently than shRNAs, pointing to the potential advantages of using gRNAs to knock out, rather than knock down, gene function in genetic screens.

While these screens in cultured cells demonstrated the power of a CRISPR-Cas9-based approach, the question remains whether similar screens can be performed in a developing organism. Excitingly, two recent proof-ofprinciple studies using worms and mice indicate that RGNs will likely be useful for in vivo genetic screens, including unbiased forward genetic screens (Liu et al. 2014a; Mashiko et al. 2014). The study in worms revealed 
that gRNA-expressing bacteria could be fed to transgenic worms expressing Cas9 to generate targeted mutations in somatic tissue and the germline, the latter giving rise to heritable mutations (Liu et al. 2014a). This approach is based on a similar method that has been widely used for RNAi-based screens in worms (Fraser et al. 2000). The study carried out in mice aimed simply to determine the frequency at which RGN-induced mutations could be recovered (Mashiko et al. 2014). This approach initially used a fluorescence reconstitution assay in HEK293T cells to eliminate ineffective gRNAs. This screening step eliminated $\sim 20 \%$ of the gRNAs, which had been randomly selected. These results point to the utility of using a cell culture-based prescreening step to rapidly enrich for gRNAs that can cleave the target site. Since the gRNAs that did not result in GFP reconstitution were not injected into mice, it is not known how performance in this in vitro assay correlates with targeting frequency in vivo. The 238 validated gRNAs, targeting 32 genes, were injected into mouse zygotes. Of the nearly 200 mice recovered, approximately half contained a targeted mutation, and $40 \%$ of the autosomal genes that were targeted had biallelic mutations. The high frequency of biallelic mutations suggests that it would be possible to screen the injected mosaic animals for phenotypes, which would preclude the necessity of generating mutant strains. Together, these studies establish the feasibility of carrying out RGN-based genetic screens in developing organisms, setting the stage for screens designed to identify new players and pathways in development.

\section{The CRISPR-Cas9 system as a platform for genome analysis}

The CRISPR-Cas9 system also provides a set of readily programmable tools to probe and manipulate the genome without altering the underlying genetic sequence. By fusing different effector domains or proteins to nuclease-inactive Cas9 (dCas9), the CRISPR-Cas9 system can be exploited to interrogate a spectrum of dynamic genome properties (Fig. 1D). The ability to use a short RNA sequence to target different dCas9 fusion proteins to a specific location within the genome has many potential applications. To date, dCas9 chimeras have been used to activate as well as repress gene expression and follow the subnuclear localization of genes and DNA sequences. Most recently, the dCas9 platform was co-opted to create another RNA-guided system for genome engineering by fusing FokI nuclease to dCas, creating an RNA-guided FokI nuclease (RFN) (Tsai et al. 2014). With the Cas9 crystal structure now in hand to provide additional insight into how Cas9 interacts with the gRNA and PAM (Jinek et al. 2014; Nishimasu et al. 2014), even more modifications and applications of this system are likely.

Catalytically inactive dCas9 was initially used as an RNA-guided platform to disrupt gene expression (Qi et al. 2013). This approach, called CRISPR interference (CRISPRi), revealed that dCas9 by itself can disrupt gene expression, most likely by sterically interfering with the transcriptional machinery. Following this work, several groups then fused different transcription activation domains, such as VP16 and VP48, to dCas9 and demonstrated that these fusion proteins can activate transgenes as well as endogenous genes (Bikard et al. 2013; Cheng et al. 2013; Maeder et al. 2013; Perez-Pinera et al. 2013; Kearns et al. 2014). Similarly, in an extension of the original CRISPRi experiments, effector domains that inhibit gene expression, such as Krüppel-associated box (KRAB) domain, have also been attached to dCas9 (Gilbert et al. 2013). While these approaches to manipulate gene expression use Cas9, it is worth pointing out that the Cas RAMP module complex (Cmr) has been developed as a tool to target mRNA for cleavage (Hale et al. 2009, 2012). Cmr belongs to the type III-B CRISPR-Cas system, which is the only CRISPR-Cas system to target RNA rather than DNA. Thus, a number of different CRISPRCas-based approaches are being developed to regulate gene expression.

dCas9 has also been co-opted to visualize the subnuclear localization of genomic sequences. By attaching a fluorescent protein to dCas9, it is possible to follow the position of repetitive telomeric sequences, satellite repeats, and individual genes within nuclei of fixed and living cells (Chen et al. 2013; Anton et al. 2014). This method is analogous to the zinc finger- and TALE-based approaches in which a fluorescent protein is joined to a programmable DNA-binding domain (Lindhout et al. 2007; Miyanari et al. 2013; Thanisch et al. 2014). By following fluorescently tagged dCas9 (or TALE or zinc finger) in living cells, it is possible to explore how genomic architecture changes as cells divide and differentiate. These approaches can also be applied to determine whether there is a functional relationship between the position of a gene (or other genetic sequence) within the nucleus and gene expression. Moreover, the TALEbased method is sensitive enough to distinguish individual single-nucleotide polymorphisms (SNPs) (Miyanari et al. 2013), allowing for allele-specific analysis.

As mentioned above, TALEs have also been used as a programmable platform. One particularly notable study created a two-component system to regulate gene expression in response to light (Konermann et al. 2013). This method, called LITE (light-inducible transcriptional effectors) capitalizes on a blue light-sensitive interaction between cryptochrome 2 (Cry2) and cryptochrome-interacting basic helix-loop-helix (CIB1). In this approach, CIB1 is fused to different transcriptional regulators, and Cry2 is targeted to specific genomic locations by attaching the TALE DNA-binding domain. LITE has been shown to effectively control gene expression in a lightdependent manner in both cultured neurons and the mouse brain. Given that the designs of the TALE and Cas9 platforms are similar, it is likely that techniques developed with one platform can be easily adapted for the other, creating an expanding number of tools to dissect gene function. One TALE-based method that has already been adapted to the dCas9 platform is the engineered DNA-binding, molecule-mediated chromatin immunoprecipitation method (or, more simply, enChIP) (Fujita 
and Fujii 2013; Fujita et al. 2013). In enChIP, a Flag-tagged TALE DNA-binding domain or dCas9 is used in combination with an anti-Flag antibody to pull down the targeted genomic sequence and associated proteins and RNA. This approach has been used to isolate telomerebinding proteins and RNAs (Fujita et al. 2013).

While these studies have demonstrated the great versatility of dCas9 and TALE platforms for genome analysis, there are several considerations that a developmental biologist should take into account before planning experiments. First, with just a few exceptions, the experiments using the dCas9 and TALE platforms have been carried out in bacteria and cultured cells. Thus, it remains to be determined how effective many of these techniques will be in dissecting genome dynamics in a developing organism. Additionally, dCas9-based approaches generally require multiple gRNAs for maximum effect; in a somewhat extreme example, it is estimated that $\sim 25-35$ gRNAs will be required to visualize the subnuclear position of a typical gene that lacks repetitive sequences (Chen et al. 2013). In addition, off-target effects are an essential consideration for both TALE and dCas9 platforms, especially given recent work revealing that dCas9 can bind up to several thousand off-target sites, many of which are in genes (Kuscu et al. 2014; Wu et al. 2014). Although these sites are infrequently cleaved by active Cas9, it is unclear how stably dCas9 associates with off-target sequences and how these interactions might affect the use of dCas9 as a programmable platform. Also, in regards to knocking down gene expression, it remains to be determined how effective CRISPRi and dCas9 chimeras are in comparison with RNAi. Notably, CRISPRi and the dCas9 chimeras designed to inhibit gene expression are reportedly less effective in cultured mammalian cells than in bacteria (Gilbert et al. 2013). Nonetheless, given the ease with which dCas9 and TALE platforms can be programmed and their versatility, the potential application of these approaches to investigating genome dynamics in vivo is enticing to consider.

\section{Concluding remarks}

RGNs have made it possible to precisely modify the genomes of a great variety of organisms and cultured cells with unprecedented ease. The rapid pace of improvements, new applications, and adoption for use in diverse organisms makes the CRISPR-Cas9 system an exciting and significant technical leap forward for developmental biology studies. Additional methodological advances will undoubtedly further enhance the use of RGNs. Currently, the majority of RGN-editing experiments have taken advantage of NHEJ to create small indels and larger deletions, which are useful for disrupting gene expression. However, to introduce specific mutations or other tailored modifications (e.g., genetically encoded tags), the HDR pathway must be activated. In most eukaryotic cells, DSBs are repaired more frequently through NHEJ than HDR (for review, see Lieber et al. 2003; Carroll 2014). Developing effective methods to bias DSB repair toward HDR to integrate a designed mutation or other modification will be of great utility for many developmental biology applications. Additional improvements in the delivery and/or expression of CRISPRCas9 components in different systems as well as in the reduction of off-target effects will further increase the efficient use of RGNs. The CRISPR-Cas9 system holds the potential to revolutionize developmental biology by making it possible to probe with exquisite control the interplay between genome activity and developmental events such as cell proliferation, differentiation, and morphogenesis.

\section{Acknowledgments}

We thank A. Mehle, D. McCulley, and members of the Wildonger laboratory for helpful comments and discussion. Work in the laboratories of M.M.H., K.M.O.-G., and J.W. is funded by startup funds from the University of Wisconsin-Madison, grants from the Wisconsin Partnership Program and March of Dimes to M.M.H., and grants from the National Institute of Neurological Disorders and Stroke, National Institutes of Health to K.M.O.-G. (R01 NS078179 and R21 NS088830) and J.W. (R00 NS072252).

\section{References}

Ammar I, Izsvak Z, Ivics Z. 2012. The Sleeping Beauty transposon toolbox. Methods Mol Biol 859: 229-240.

Ansai S, Kinoshita M. 2014. Targeted mutagenesis using CRISPR/Cas system in medaka. Biol Open 3: 362-371.

Anton T, Bultmann S, Leonhardt H, Markaki Y. 2014. Visualization of specific DNA sequences in living mouse embryonic stem cells with a programmable fluorescent CRISPR/Cas system. Nucleus 5: 163-172.

Auer TO, Duroure K, De Cian A, Concordet JP, Del Bene F. 2014. Highly efficient CRISPR/Cas9-mediated knock-in in zebrafish by homology-independent DNA repair. Genome Res 24: 142-153.

Banaszynski LA, Chen LC, Maynard-Smith LA, Ooi AG, Wandless TJ. 2006. A rapid, reversible, and tunable method to regulate protein function in living cells using synthetic small molecules. Cell 126: 995-1004.

Barrangou R, Fremaux C, Deveau H, Richards M, Boyaval P, Moineau S, Romero DA, Horvath P. 2007. CRISPR provides acquired resistance against viruses in prokaryotes. Science 315: 1709-1712.

Bassett AR, Liu JL. 2014. CRISPR/Cas9 and genome editing in Drosophila. J Genet Genomics 41: 7-19.

Belhaj K, Chaparro-Garcia A, Kamoun S, Nekrasov V. 2013. Plant genome editing made easy: targeted mutagenesis in model and crop plants using the CRISPR/Cas system. Plant Methods 9: 39.

Bibikova M, Golic M, Golic KG, Carroll D. 2002. Targeted chromosomal cleavage and mutagenesis in Drosophila using zinc-finger nucleases. Genetics 161: 1169-1175.

Bikard D, Jiang W, Samai P, Hochschild A, Zhang F, Marraffini LA. 2013. Programmable repression and activation of bacterial gene expression using an engineered CRISPR-Cas system. Nucleic Acids Res 41: 7429-7437.

Blitz IL, Biesinger J, Xie X, Cho KW. 2013. Biallelic genome modification in $\mathrm{F}(0)$ Xenopus tropicalis embryos using the CRISPR/Cas system. Genesis 51: 827-834.

Boissel S, Jarjour J, Astrakhan A, Adey A, Gouble A, Duchateau P, Shendure J, Stoddard BL, Certo MT, Baker D, et al. 2014. megaTALs: a rare-cleaving nuclease architecture for therapeutic genome engineering. Nucleic Acids Res 42: 2591-2601. 
Bolotin A, Quinquis B, Sorokin A, Ehrlich SD. 2005. Clustered regularly interspaced short palindrome repeats (CRISPRs) have spacers of extrachromosomal origin. Microbiology 151: 2551-2561.

Capecchi MR. 2005. Gene targeting in mice: functional analysis of the mammalian genome for the twenty-first century. Nat Rev Genet 6: 507-512.

Carroll D. 2011. Genome engineering with zinc-finger nucleases. Genetics 188: 773-782.

Carroll D. 2014. Genome engineering with targetable nucleases. Annu Rev Biochem 83: 409-439.

Chang N, Sun C, Gao L, Zhu D, Xu X, Zhu X, Xiong JW, Xi JJ. 2013. Genome editing with RNA-guided Cas9 nuclease in zebrafish embryos. Cell Res 23: 465-472.

Chen B, Gilbert LA, Cimini BA, Schnitzbauer J, Zhang W, Li GW, Park J, Blackburn EH, Weissman JS, Qi LS, et al. 2013. Dynamic imaging of genomic loci in living human cells by an optimized CRISPR/Cas system. Cell 155: 1479-1491.

Chen H, Choi J, Bailey S. 2014. Cut site selection by the two nuclease domains of the Cas9 RNA-guided endonuclease. I Biol Chem 289: 13284-13294.

Cheng AW, Wang H, Yang H, Shi L, Katz Y, Theunissen TW, Rangarajan S, Shivalila CS, Dadon DB, Jaenisch R. 2013. Multiplexed activation of endogenous genes by CRISPR-on, an RNA-guided transcriptional activator system. Cell Res 23: 1163-1171.

Cho SW, Kim S, Kim Y, Kweon J, Kim HS, Bae S, Kim JS. 2014. Analysis of off-target effects of CRISPR/Cas-derived RNAguided endonucleases and nickases. Genome Res 24: 132141.

Choi PS, Meyerson M. 2014. Targeted genomic rearrangements using CRISPR/Cas technology. Nat Commun 5: 3728.

Cong L, Ran FA, Cox D, Lin S, Barretto R, Habib N, Hsu PD, Wu X, Jiang W, Marraffini LA, et al. 2013. Multiplex genome engineering using CRISPR/Cas systems. Science 339: 819823.

Daimon T, Kiuchi T, Takasu Y. 2014. Recent progress in genome engineering techniques in the silkworm, Bombyx mori. Dev Growth Differ 56: 14-25.

Dean KM, Palmer AE. 2014. Advances in fluorescence labeling strategies for dynamic cellular imaging. Nat Chem Biol 10: 512-523.

Deltcheva E, Chylinski K, Sharma CM, Gonzales K, Chao Y, Pirzada ZA, Eckert MR, Vogel J, Charpentier E. 2011. CRISPR RNA maturation by trans-encoded small RNA and host factor RNase III. Nature 471: 602-607.

Ding Q, Regan SN, Xia Y, Oostrom LA, Cowan CA, Musunuru K. 2013. Enhanced efficiency of human pluripotent stem cell genome editing through replacing TALENs with CRISPRs. Cell Stem Cell 12: 393-394.

Duan J, Lu G, Xie Z, Lou M, Luo J, Guo L, Zhang Y. 2014. Genome-wide identification of CRISPR/Cas9 off-targets in human genome. Cell Res 24: 1009-1012.

Esvelt KM, Mali P, Braff JL, Moosburner M, Yaung SJ, Church GM. 2013. Orthogonal Cas9 proteins for RNA-guided gene regulation and editing. Nat Methods 10: 1116-1121.

Fauser F, Schiml S, Puchta H. 2014. Both CRISPR/Cas-based nucleases and nickases can be used efficiently for genome engineering in Arabidopsis thaliana. Plant J 79: 348-359.

Feng Z, Zhang B, Ding W, Liu X, Yang DL, Wei P, Cao F, Zhu S, Zhang F, Mao Y, et al. 2013. Efficient genome editing in plants using a CRISPR/Cas system. Cell Res 23: 1229-1232.

Flowers GP, Timberlake AT, McLean KC, Monaghan JR, Crews CM. 2014. Highly efficient targeted mutagenesis in axolotl using Cas9 RNA-guided nuclease. Development 141: 21652171.
Fraser AG, Kamath RS, Zipperlen P, Martinez-Campos M, Sohrmann M, Ahringer J. 2000. Functional genomic analysis of C. elegans chromosome I by systematic RNA interference. Nature 408: 325-330.

Friedel RH, Soriano P. 2010. Gene trap mutagenesis in the mouse. Methods Enzymol 477: 243-269.

Fu Y, Sander JD, Reyon D, Cascio VM, Joung JK. 2014. Improving CRISPR-Cas nuclease specificity using truncated guide RNAs. Nat Biotechnol 32: 279-284.

Fujii W, Onuma A, Sugiura K, Naito K. 2014. Efficient generation of genome-modified mice via offset-nicking by CRISPR/Cas system. Biochem Biophys Res Commun 445: 791-794.

Fujita T, Fujii H. 2013. Efficient isolation of specific genomic regions and identification of associated proteins by engineered DNA-binding molecule-mediated chromatin immunoprecipitation (enChIP) using CRISPR. Biochem Biophys Res Commun 439: 132-136.

Fujita T, Asano Y, Ohtsuka J, Takada Y, Saito K, Ohki R, Fujii H. 2013. Identification of telomere-associated molecules by engineered DNA-binding molecule-mediated chromatin immunoprecipitation (enChIP). Sci Rep 3: 3171.

Gao Y, Zhao Y. 2014. Self-processing of ribozyme-flanked RNAs into guide RNAs in vitro and in vivo for CRISPR-mediated genome editing. J Integr Plant Biol 56: 343-349.

Gasiunas G, Barrangou R, Horvath P, Siksnys V. 2012. Cas9crRNA ribonucleoprotein complex mediates specific DNA cleavage for adaptive immunity in bacteria. Proc Natl Acad Sci 109: E2579-E2586.

Gilbert LA, Larson MH, Morsut L, Liu Z, Brar GA, Torres SE, Stern-Ginossar N, Brandman O, Whitehead EH, Doudna JA, et al. 2013. CRISPR-mediated modular RNA-guided regulation of transcription in eukaryotes. Cell 154: 442-451.

Gonzalez F, Zhu Z, Shi ZD, Lelli K, Verma N, Li QV, Huangfu D. 2014. An iCRISPR platform for rapid, multiplexable, and inducible genome editing in human pluripotent stem cells. Cell Stem Cell doi: 10.1016/j.stem.2014.05.018.

Gratz SI, Wildonger J, Harrison MM, O'Connor-Giles KM. 2013. CRISPR/Cas9-mediated genome engineering and the promise of designer flies on demand. Fly (Austin) 7: 249-255.

Gratz SJ, Ukken FP, Rubinstein CD, Thiede G, Donohue LK, Cummings AM, O'Connor-Giles KM. 2014. Highly specific and efficient CRISPR/Cas9-catalyzed homology-directed repair in Drosophila. Genetics 196: 961-971.

Guo X, Zhang T, Hu Z, Zhang Y, Shi Z, Wang Q, Cui Y, Wang F, Zhao H, Chen Y. 2014. Efficient RNA/Cas9-mediated genome editing in Xenopus tropicalis. Development 141: 707714.

Hai T, Teng F, Guo R, Li W, Zhou Q. 2014. One-step generation of knockout pigs by zygote injection of CRISPR/Cas system. Cell Res 24: 372-375.

Hale CR, Zhao P, Olson S, Duff MO, Graveley BR, Wells L, Terns RM, Terns MP. 2009. RNA-guided RNA cleavage by a CRISPR RNA-Cas protein complex. Cell 139: 945-956.

Hale CR, Majumdar S, Elmore J, Pfister N, Compton M, Olson S, Resch AM, Glover CV 3rd, Graveley BR, Terns RM, et al. 2012. Essential features and rational design of CRISPR RNAs that function with the Cas RAMP module complex to cleave RNAs. Mol Cell 45: 292-302.

Hockemeyer D, Jaenisch R. 2010. Gene targeting in human pluripotent cells. Cold Spring Harb Symp Quant Biol 75: 201-209.

Hou Z, Zhang Y, Propson NE, Howden SE, Chu LF, Sontheimer EJ, Thomson JA. 2013. Efficient genome engineering in human pluripotent stem cells using Cas9 from Neisseria meningitidis. Proc Natl Acad Sci 110: 15644-15649. 
Hsu PD, Scott DA, Weinstein JA, Ran FA, Konermann S, Agarwala V, Li Y, Fine EJ, Wu X, Shalem O, et al. 2013. DNA targeting specificity of RNA-guided Cas9 nucleases. Nat Biotechnol 31: 827-832.

Hsu PD, Lander ES, Zhang F. 2014. Development and applications of CRISPR-Cas9 for genome engineering. Cell 157: $1262-1278$.

Hwang WY, Fu Y, Reyon D, Maeder ML, Tsai SQ, Sander JD, Peterson RT, Yeh JR, Joung JK. 2013. Efficient genome editing in zebrafish using a CRISPR-Cas system. Nat Biotechnol 31: 227-229.

Ishino $\mathrm{Y}$, Shinagawa $\mathrm{H}$, Makino K, Amemura M, Nakata A. 1987. Nucleotide sequence of the iap gene, responsible for alkaline phosphatase isozyme conversion in Escherichia coli, and identification of the gene product. J Bacteriol 169: 54295433.

Jao LE, Wente SR, Chen W. 2013. Efficient multiplex biallelic zebrafish genome editing using a CRISPR nuclease system. Proc Natl Acad Sci 110: 13904-13909.

Jia H, Wang N. 2014. targeted genome editing of sweet orange using Cas9/sgRNA. PLoS ONE 9: e93806.

Jiang W, Bikard D, Cox D, Zhang F, Marraffini LA. 2013a. RNAguided editing of bacterial genomes using CRISPR-Cas systems. Nat Biotechnol 31: 233-239.

Jiang W, Zhou H, Bi H, Fromm M, Yang B, Weeks DP. 2013b. Demonstration of CRISPR/Cas9/sgRNA-mediated targeted gene modification in Arabidopsis, tobacco, sorghum and rice. Nucleic Acids Res 41: e188.

Jinek M, Chylinski K, Fonfara I, Hauer M, Doudna JA, Charpentier E. 2012. A programmable dual-RNA-guided DNA endonuclease in adaptive bacterial immunity. Science 337: 816-821.

Jinek M, East A, Cheng A, Lin S, Ma E, Doudna J. 2013. RNAprogrammed genome editing in human cells. eLife 2: e00471.

Jinek M, Jiang F, Taylor DW, Sternberg SH, Kaya E, Ma E, Anders C, Hauer M, Zhou K, Lin S, et al. 2014. Structures of Cas9 endonucleases reveal RNA-mediated conformational activation. Science 343: 1247997.

Joung JK, Sander JD. 2013. TALENs: a widely applicable technology for targeted genome editing. Nat Rev Mol Cell Biol 14: 49-55.

Kearns NA, Genga RM, Enuameh MS, Garber M, Wolfe SA, Maehr R. 2014. Cas9 effector-mediated regulation of transcription and differentiation in human pluripotent stem cells. Development 141: 219-223.

Kiskinis E, Sandoe J, Williams LA, Boulting GL, Moccia R, Wainger BJ, Han S, Peng T, Thams S, Mikkilineni S, et al. 2014. Pathways disrupted in human ALS motor neurons identified through genetic correction of mutant SOD1. Cell Stem Cell 14: 781-795.

Koike-Yusa H, Li Y, Tan EP, Velasco-Herrera Mdel C, Yusa K. 2014. Genome-wide recessive genetic screening in mammalian cells with a lentiviral CRISPR-guide RNA library. Nat Biotechnol 32: 267-273.

Kondo S, Ueda R. 2013. Highly improved gene targeting by germline-specific Cas9 expression in Drosophila. Genetics 195: 715-721.

Konermann S, Brigham MD, Trevino AE, Hsu PD, Heidenreich M, Cong L, Platt RJ, Scott DA, Church GM, Zhang F. 2013. Optical control of mammalian endogenous transcription and epigenetic states. Nature 500: 472-476.

Kuscu C, Arslan S, Singh R, Thorpe J, Adli M. 2014. Genomewide analysis reveals characteristics of off-target sites bound by the Cas9 endonuclease. Nat Biotechnol 32: 677683.
Li D, Qiu Z, Shao Y, Chen Y, Guan Y, Liu M, Li Y, Gao N, Wang $\mathrm{L}$, Lu X, et al. 2013a. Heritable gene targeting in the mouse and rat using a CRISPR-Cas system. Nat Biotechnol 31: 681683.

Li W, Teng F, Li T, Zhou Q. 2013b. Simultaneous generation and germline transmission of multiple gene mutations in rat using CRISPR-Cas systems. Nat Biotechnol 31: 684-686.

Li M, Yang H, Zhao J, Fang L, Shi H, Li M, Sun Y, Zhang X, Jiang D, Zhou L et al. 2014a. Efficient and heritable gene targeting in tilapia by CRISPR/Cas9. Genetics 197: 591-599.

Li W, Li X, Li T, Jiang MG, Wan H, Luo GZ, Feng C, Cui X, Teng F, Yuan Y, et al. 2014b. Genetic modification and screening in rat using haploid embryonic stem cells. Cell Stem Cell 14: 404-414.

Liang Z, Zhang K, Chen K, Gao C. 2014. Targeted mutagenesis in Zea mays using TALENs and the CRISPR/Cas system. J Genet Genomics 41: 63-68.

Lieber MR, Ma Y, Pannicke U, Schwarz K. 2003. Mechanism and regulation of human non-homologous DNA end-joining. Nat Rev Mol Cell Biol 4: 712-720.

Lin Y, Cradick TJ, Brown MT, Deshmukh H, Ranjan P, Sarode N, Wile BM, Vertino PM, Stewart FJ, Bao G. 2014. CRISPR/Cas9 systems have off-target activity with insertions or deletions between target DNA and guide RNA sequences. Nucleic Acids Res 42: 7473-7485.

Lindhout BI, Fransz P, Tessadori F, Meckel T, Hooykaas PJ, van der Zaal BJ. 2007. Live cell imaging of repetitive DNA sequences via GFP-tagged polydactyl zinc finger proteins. Nucleic Acids Res 35: e107.

Lisa Li H, Nakano T, Hotta A. 2014. Genetic correction using engineered nucleases for gene therapy applications. Dev Growth Differ 56: 63-77.

Liu P, Long L, Xiong K, Yu B, Chang N, Xiong JW, Zhu Z, Liu D. 2014a. Heritable/conditional genome editing in C. elegans using a CRISPR-Cas9 feeding system. Cell Res 24: 886-889.

Liu Y, Ma S, Wang X, Chang J, Gao J, Shi R, Zhang J, Lu W, Liu Y, Zhao $P$, et al. 2014b. Highly efficient multiplex targeted mutagenesis and genomic structure variation in Bombyx mori cells using CRISPR/Cas9. Insect Biochem Mol Biol 49: $35-42$.

Ma S, Chang J, Wang X, Liu Y, Zhang J, Lu W, Gao J, Shi R, Zhao $\mathrm{P}$, Xia Q 2014a. CRISPR/Cas9 mediated multiplex genome editing and heritable mutagenesis of BmKu70 in Bombyx mori. Sci Rep 4: 4489.

Ma Y, Ma J, Zhang X, Chen W, Yu L, Lu Y, Bai L, Shen B, Huang $\mathrm{X}$, Zhang L. 2014b. Generation of eGFP and Cre knockin rats by CRISPR/Cas9. FEBS J doi: 10.1111/febs.12935.

Ma Y, Shen B, Zhang X, Lu Y, Chen W, Ma J, Huang X, Zhang L. 2014c. Heritable multiplex genetic engineering in rats using CRISPR/Cas9. PLOS ONE 9: e89413.

Ma Y, Zhang X, Shen B, Lu Y, Chen W, Ma J, Bai L, Huang X, Zhang L. 2014d. Generating rats with conditional alleles using CRISPR/Cas9. Cell Res 24: 122-125.

Maeder ML, Linder SJ, Cascio VM, Fu Y, Ho QH, Joung JK. 2013. CRISPR RNA-guided activation of endogenous human genes. Nat Methods 10: 977-979.

Mali P, Aach J, Stranges PB, Esvelt KM, Moosburner M, Kosuri S, Yang L, Church GM. 2013a. CAS9 transcriptional activators for target specificity screening and paired nickases for cooperative genome engineering. Nat Biotechnol 31: 833838.

Mali P, Esvelt KM, Church GM. 2013b. Cas9 as a versatile tool for engineering biology. Nat Methods 10: 957-963.

Mali P, Yang L, Esvelt KM, Aach J, Guell M, DiCarlo JE, Norville JE, Church GM. 2013c. RNA-guided human genome engineering via Cas9. Science 339: 823-826. 
Mansour SL, Thomas KR, Capecchi MR. 1988. Disruption of the proto-oncogene int-2 in mouse embryo-derived stem cells: a general strategy for targeting mutations to non-selectable genes. Nature 336: 348-352.

Mao Y, Zhang H, Xu N, Zhang B, Gou F, Zhu JK. 2013. Application of the CRISPR-Cas system for efficient genome engineering in plants. Mol Plant 6: 2008-2011.

Mashiko D, Young SA, Muto M, Kato H, Nozawa K, Ogawa M, Noda T, Kim YJ, Satouh Y, Fujihara Y, et al. 2014. Feasibility for a large scale mouse mutagenesis by injecting CRISPR/ Cas plasmid into zygotes. Dev Growth Differ 56: 122-129.

Miyanari Y, Ziegler-Birling C, Torres-Padilla ME. 2013. Live visualization of chromatin dynamics with fluorescent TALEs. Nat Struct Mol Biol 20: 1321-1324.

Mohr S, Bakal C, Perrimon N. 2010. Genomic screening with RNAi: results and challenges. Annu Rev Biochem 79: 37-64.

Mojica FJ, Diez-Villasenor C, Garcia-Martinez J, Soria E. 2005. Intervening sequences of regularly spaced prokaryotic repeats derive from foreign genetic elements. I Mol Evol 60: 174-182.

Nakanishi T, Kato Y, Matsuura T, Watanabe H. 2014. CRISPR/ Cas-mediated targeted mutagenesis in Daphnia magna. PLOS ONE 9: e98363.

Nakayama T, Fish MB, Fisher M, Oomen-Hajagos J, Thomsen GH, Grainger RM. 2013. Simple and efficient CRISPR/Cas9mediated targeted mutagenesis in Xenopus tropicalis. Genesis 51: 835-843.

Nishimasu H, Ran FA, Hsu PD, Konermann S, Shehata SI, Dohmae N, Ishitani R, Zhang F, Nureki O. 2014. Crystal structure of Cas9 in complex with guide RNA and target DNA. Cell 156: 935-949.

Nissim L, Perli SD, Fridkin A, Perez-Pinera P, Lu TK. 2014. Multiplexed and programmable regulation of gene networks with an integrated RNA and CRISPR/Cas toolkit in human cells. Mol Cell 54: 698-710.

Niu J, Zhang B, Chen H. 2014a. Applications of TALENs and CRISPR/Cas9 in human cells and their potentials for gene therapy. Mol Biotechnol 56: 681-688.

Niu Y, Shen B, Cui Y, Chen Y, Wang J, Wang L, Kang Y, Zhao X, Si W, Li W, et al. 2014b. Generation of gene-modified cynomolgus monkey via Cas9/RNA-mediated gene targeting in one-cell embryos. Cell 156: 836-843.

Park A, Won ST, Pentecost M, Bartkowski W, Lee B. 2014. CRISPR/Cas9 allows efficient and complete knock-in of a destabilization domain-tagged essential protein in a human cell line, allowing rapid knockdown of protein function. PLOS ONE 9: e95101.

Perez-Pinera P, Kocak DD, Vockley CM, Adler AF, Kabadi AM, Polstein LR, Thakore PI, Glass KA, Ousterout DG, Leong $\mathrm{KW}$, et al. 2013. RNA-guided gene activation by CRISPRCas9-based transcription factors. Nat Methods 10: 973-976.

Port F, Chen HM, Lee T, Bullock SL. 2014. Optimized CRISPR/ Cas tools for efficient germline and somatic genome engineering in Drosophila. Proc Natl Acad Sci 111: E2967-E2976.

Pourcel C, Salvignol G, Vergnaud G. 2005. CRISPR elements in Yersinia pestis acquire new repeats by preferential uptake of bacteriophage DNA, and provide additional tools for evolutionary studies. Microbiology 151: 653-663.

Qi LS, Larson MH, Gilbert LA, Doudna JA, Weissman JS, Arkin AP, Lim WA. 2013. Repurposing CRISPR as an RNA-guided platform for sequence-specific control of gene expression. Cell 152: 1173-1183.

Ran FA, Hsu PD, Lin CY, Gootenberg JS, Konermann S, Trevino AE, Scott DA, Inoue A, Matoba S, Zhang Y, et al. 2013. Double nicking by RNA-guided CRISPR Cas9 for enhanced genome editing specificity. Cell 154: 1380-1389.
Ren X, Sun J, Housden BE, Hu Y, Roesel C, Lin S, Liu LP, Yang Z, Mao D, Sun L, et al. 2013. Optimized gene editing technology for Drosophila melanogaster using germ line-specific Cas9. Proc Natl Acad Sci 110: 19012-19017.

Rong Z, Zhu S, Xu Y, Fu X. 2014. Homologous recombination in human embryonic stem cells using CRISPR/Cas9 nickase and a long DNA donor template. Protein Cell 5: 258-260.

Sander JD, Joung JK. 2014. CRISPR-Cas systems for editing, regulating and targeting genomes. Nat Biotechnol 32: 347-355.

Sato M, Miyoshi K, Nagao Y, Nishi Y, Ohtsuka M, Nakamura S, Sakurai T, Watanabe S. 2014. The combinational use of CRISPR/Cas9-based gene editing and targeted toxin technology enables efficient biallelic knockout of the $\alpha-1,3$ galactosyltransferase gene in porcine embryonic fibroblasts. Xenotransplantation 21: 291-300.

Schwank G, Koo BK, Sasselli V, Dekkers JF, Heo I, Demircan T, Sasaki N, Boymans S, Cuppen E, van der Ent CK, et al. 2013. Functional repair of CFTR by CRISPR/Cas9 in intestinal stem cell organoids of cystic fibrosis patients. Cell Stem Cell 13: $653-658$.

Sebo ZL, Lee HB, Peng Y, Guo Y. 2014. A simplified and efficient germline-specific CRISPR/Cas9 system for Drosophila genomic engineering. Fly (Austin) 8: 52-57.

Shalem O, Sanjana NE, Hartenian E, Shi X, Scott DA, Mikkelsen TS, Heckl D, Ebert BL, Root DE, Doench JG, et al. 2014. Genome-scale CRISPR-Cas9 knockout screening in human cells. Science 343: 84-87.

Shen B, Zhang W, Zhang J, Zhou J, Wang J, Chen L, Wang L, Hodgkins A, Iyer V, Huang X, et al. 2014. Efficient genome modification by CRISPR-Cas9 nickase with minimal offtarget effects. Nat Methods 11: 399-402.

Skarnes WC, Rosen B, West AP, Koutsourakis M, Bushell W, Iyer V, Mujica AO, Thomas M, Harrow J, Cox T, et al. 2011. A conditional knockout resource for the genome-wide study of mouse gene function. Nature 474: 337-342.

Smith C, Gore A, Yan W, Abalde-Atristain L, Li Z, He C, Wang Y, Brodsky RA, Zhang K, Cheng L, et al. 2014. Wholegenome sequencing analysis reveals high specificity of CRISPR/Cas9 and TALEN-based genome editing in human iPSCs. Cell Stem Cell 15: 12-13.

Smithies O, Gregg RG, Boggs SS, Koralewski MA, Kucherlapati RS. 1985. Insertion of DNA sequences into the human chromosomal $\beta$-globin locus by homologous recombination. Nature 317: 230-234.

Sorek R, Lawrence CM, Wiedenheft B. 2013. CRISPR-mediated adaptive immune systems in bacteria and archaea. Annu Rev Biochem 82: 237-266.

St Johnston D. 2013. Using mutants, knockdowns, and transgenesis to investigate gene function in Drosophila. Wiley Interdiscip Rev Dev Biol 2: 587-613.

Struhl K, Segal E. 2013. Determinants of nucleosome positioning. Nat Struct Mol Biol 20: 267-273.

Sugano SS, Shirakawa M, Takagi J, Matsuda Y, Shimada T, HaraNishimura I, Kohchi T. 2014. CRISPR/Cas9-mediated targeted mutagenesis in the liverwort Marchantia polymorpha L. Plant Cell Physiol 55: 475-481.

Suzuki K, Yu C, Qu J, Li M, Yao X, Yuan T, Goebl A, Tang S, Ren R, Aizawa E, et al. 2014. Targeted gene correction minimally impacts whole-genome mutational load in human-diseasespecific induced pluripotent stem cell clones. Cell Stem Cell 15: 31-36.

Terns RM, Terns MP. 2014. CRISPR-based technologies: prokaryotic defense weapons repurposed. Trends Genet 30: 111118.

Thanisch K, Schneider K, Morbitzer R, Solovei I, Lahaye T, Bultmann S, Leonhardt H. 2014. Targeting and tracing of 
specific DNA sequences with dTALEs in living cells. Nucleic Acids Res 42: e38.

Thomas KR, Folger KR, Capecchi MR. 1986. High frequency targeting of genes to specific sites in the mammalian genome. Cell 44: 419-428.

Torres R, Martin MC, Garcia A, Cigudosa JC, Ramirez JC, Rodriguez-Perales S. 2014. Engineering human tumour-associated chromosomal translocations with the RNA-guided CRISPR-Cas9 system. Nat Commun 5: 3964.

Tsai SQ, Wyvekens N, Khayter C, Foden JA, Thapar V, Reyon D, Goodwin MJ, Aryee MJ, Joung JK. 2014. Dimeric CRISPR RNA-guided FokI nucleases for highly specific genome editing. Nat Biotechnol 32: 569-576.

Upadhyay SK, Kumar J, Alok A, Tuli R. 2013. RNA-guided genome editing for target gene mutations in wheat. G3 3: 2233-2238.

Veres A, Gosis BS, Ding Q, Collins R, Ragavendran A, Brand H, Erdin S, Talkowski ME, Musunuru K. 2014. Low incidence of off-target mutations in individual CRISPR-Cas9 and TALEN targeted human stem cell clones detected by whole-genome sequencing. Cell Stem Cell 15: 27-30.

Waaijers S, Boxem M 2014. Engineering the Caenorhabditis elegans genome with CRISPR/Cas9. Methods 68: 381-388.

Wang H, Yang H, Shivalila CS, Dawlaty MM, Cheng AW, Zhang F, Jaenisch R. 2013a. One-step generation of mice carrying mutations in multiple genes by CRISPR/Cas-mediated genome engineering. Cell 153: 910-918.

Wang Y, Li Z, Xu J, Zeng B, Ling L, You L, Chen Y, Huang Y, Tan A. 2013b. The CRISPR/Cas system mediates efficient genome engineering in Bombyx mori. Cell Res 23: 14141416.

Wang T, Wei JJ, Sabatini DM, Lander ES. 2014. Genetic screens in human cells using the CRISPR-Cas9 system. Science 343: 80-84.

Wei W, Xin H, Roy B, Dai J, Miao Y, Gao G. 2014. Heritable genome editing with CRISPR/Cas9 in the silkworm, Bombyx mori. PLOS ONE 9: e101210.

Wilkinson R, Wiedenheft B. 2014. A CRISPR method for genome engineering. F1000Prime Rep 6: 3.

Wu Y, Liang D, Wang Y, Bai M, Tang W, Bao S, Yan Z, Li D, Li J. 2013. Correction of a genetic disease in mouse via use of CRISPR-Cas9. Cell Stem Cell 13: 659-662.

Wu X, Scott DA, Kriz AJ, Chiu AC, Hsu PD, Dadon DB, Cheng AW, Trevino AE, Konermann S, Chen S, et al. 2014. Genomewide binding of the CRISPR endonuclease Cas9 in mammalian cells. Nat Biotechnol 32: 670-676.

Xue Z, Ren M, Wu M, Dai J, Rong YS, Gao G. 2014. Efficient gene knock-out and knock-in with transgenic Cas9 in Drosophila. G3 4: 925-929.

Yang H, Wang H, Shivalila CS, Cheng AW, Shi L, Jaenisch R. 2013. One-step generation of mice carrying reporter and conditional alleles by CRISPR/Cas-mediated genome engineering. Cell 154: 1370-1379.

Yang D, Xu J, Zhu T, Fan J, Lai L, Zhang J, Chen YE. 2014. Effective gene targeting in rabbits using RNA-guided Cas9 nucleases. J Mol Cell Biol 6: 97-99.

Yasue A, Mitsui SN, Watanabe T, Sakuma T, Oyadomari $\mathrm{S}$, Yamamoto T, Noji S, Mito T, Tanaka E. 2014. Highly efficient targeted mutagenesis in one-cell mouse embryos mediated by the TALEN and CRISPR/Cas systems. Sci Rep 4: 5705.

Yen ST, Zhang M, Deng JM, Usman SJ, Smith CN, ParkerThornburg J, Swinton PG, Martin JF, Behringer RR. 2014. Somatic mosaicism and allele complexity induced by CRISPR/Cas9 RNA injections in mouse zygotes. Dev Biol doi: 10.1016/j.ydbio.2014.06.017.
Yin H, Xue W, Chen S, Bogorad RL, Benedetti E, Grompe M, Koteliansky V, Sharp PA, Jacks T, Anderson DG. 2014. Genome editing with Cas9 in adult mice corrects a disease mutation and phenotype. Nat Biotechnol 32: 551-553.

Yu Z, Ren M, Wang Z, Zhang B, Rong YS, Jiao R, Gao G. 2013. Highly efficient genome modifications mediated by CRISPR/ Cas9 in Drosophila. Genetics 195: 289-291.

Yu Z, Chen H, Liu J, Zhang H, Yan Y, Zhu N, Guo Y, Yang B, Chang Y, Dai F, et al. 2014. Various applications of TALENand CRISPR/Cas9-mediated homologous recombination to modify the Drosophila genome. Biol Open 3: 271-280.

Zhang Y, Ge X, Yang F, Zhang L, Zheng J, Tan X, Jin ZB, Qu J, Gu F. 2014. Comparison of non-canonical PAMs for CRISPR/ Cas9-mediated DNA cleavage in human cells. Sci Rep 4: 5405.

Zhou Y, Zhu S, Cai C, Yuan P, Li C, Huang Y, Wei W. 2014. High-throughput screening of a CRISPR/Cas9 library for functional genomics in human cells. Nature 509: 487-491. 


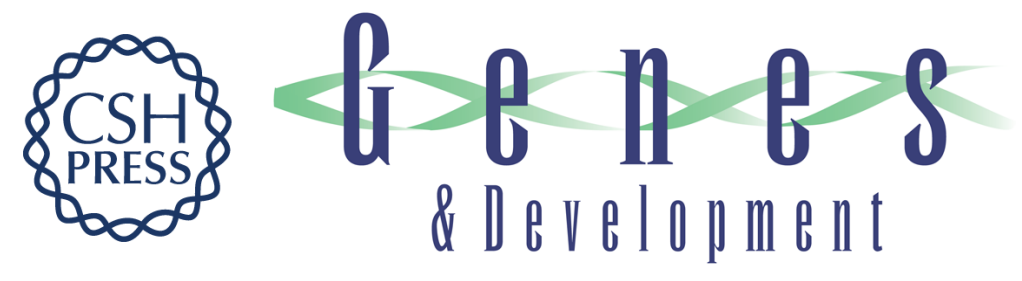

\section{A CRISPR view of development}

Melissa M. Harrison, Brian V. Jenkins, Kate M. O'Connor-Giles, et al.

Genes Dev. 2014, 28:

Access the most recent version at doi:10.1101/gad.248252.114

References This article cites 146 articles, 30 of which can be accessed free at: http://genesdev.cshlp.org/content/28/17/1859.full.html\#ref-list-1

Creative This article, published in Genes \& Development, is available under a Creative Commons

Commons License (Attribution-NonCommercial 4.0 International), as described at

License http://creativecommons.org/licenses/by-nc/4.0/.

Email Alerting Receive free email alerts when new articles cite this article - sign up in the box at the top Service right corner of the article or click here.

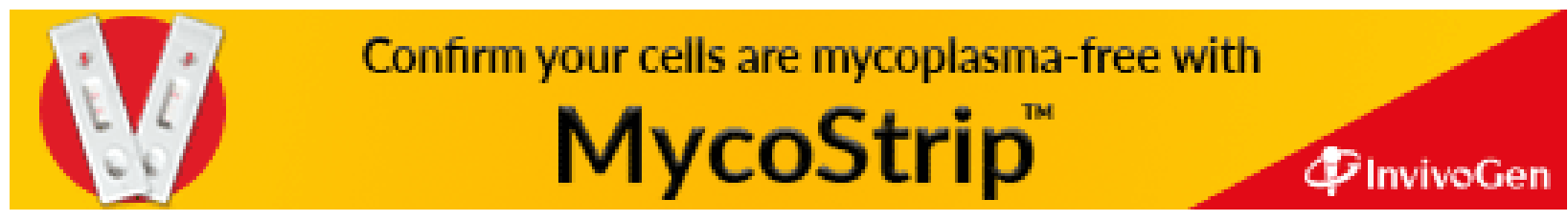

